\title{
ROAD TRAFFIC MEASUREMENT AND RELATED DATA FUSION METHODOLOGY FOR TRAFFIC ESTIMATION
}

\author{
Tamás Tettamanti ${ }^{1}$, MártonTamás Horváth ${ }^{2}$, István Varga ${ }^{3}$ \\ Department of Control for Transportation and Vehicle Systems, \\ Budapest University of Technology and Economics, \\ Stoczek J. u. 2., H-1111 Budapest, Hungary \\ E-mail: ${ }^{1}$ tettamanti@mail.bme.hu, ${ }^{2}$ hmt1990@gmail.com, ${ }^{3}$ ivarga@mail.bme.hu
}

\begin{abstract}
The knowledge of road traffic parameters is of crucial importance to ensure state-of-the-art traffic services either in public or private transport. In our days, a plethora of road traffic data are continuously collected producing historical and real-time traffic information as well. The available information, however, arrive from inhomogeneous sensor systems. Therefore, a data fusion methodology is proposed based on Switching Kalman Filter. The concept enables efficient travel time estimation for urban road traffic network. On the other hand, the method may contribute to a better macroscopic traffic modelling.
\end{abstract}

Keywords: Road Traffic Estimation, Data fusion, Switching Kalman Filter

\section{Introduction}

Planning schedules of urban bus services or the control of signalized junctions can be based on the knowledge of relevant traffic data. In possession of real-time traffic information, a wide range of new traffic services can be introduced, e.g. route planning for private travellers, energy-efficient vehicle control or optimal control of the whole traffic network. However, completely coherent information of the traffic state cannot be provided only by different types of sensor data that are analysed separately. This way network parameters, such as volume, density, travel time or origin-destination (OD) data cannot be determined within the whole traffic network. Covering the whole network by sensors would be the easiest method for gathering data about its traffic state. At the same time, this is only a theoretic solution, since installation and maintenance of required sensors would be extremely expensive. Therefore, a costefficient method is necessary to realize a cost efficient traffic state estimation based on rarely located sensors producing different types of data. Certainly, developing an algorithm that meets these requirements has several difficulties caused by inhomogeneous data types, e.g. different semantics, difference in time and location and data dropout. Considering all of these factors, the purpose of this article is the evaluation of state-of-the-art measurement data in order to fuse them. The basic idea is that the fusion of information pieces produced by different types of sensors can result in better and more efficient traffic services and systems compared to those techniques that deal with different types of sensors separately.

In the last few decades, information technology has developed rapidly; several new technologies have been introduced. Some of them are just in their beginning steps. Mobile phones (that are used by nearly all adult citizens), widespread and free smartphone applications, fleet management systems (on public transport and private vehicles as well) and several other techniques make it possible to realize a state-of-the-art and cost-efficient service.

\section{Measurement techniques and their features}

Measurement systems applicable to road traffic data collection can be divided into two parts: traditional and alternative techniques. Traditional techniques are sensor technologies that have been developed to measure road traffic parameters. These devices are e.g. loop-detector, magnetic detector and camera. Unlike the previous ones, alternative technologies have not been originally developed to measure road traffic parameters, even though they can provide these types of information, e.g. fleet management systems, cellular and GPS data of mobile telephones, etc. The features of different measurement types are described in this section. 


\subsection{Traditional road traffic measurement technologies}

Traditional road traffic sensors are directly planned for accurate traffic data measurement. Basically, cross sectional measurements can be done on the traffic road network (Ludvig et al., 2012). Therefore, time dependent parameters can be obtained. These are time occupancy, time headway, volume and time mean speed. By using a single detector, time mean speed can only be estimated (by calculating with a mean vehicle length). If more detectors are placed after each other within a short distance (usually on motorways), time mean speed can be measured, not just estimated, therefore the results are more precise. Even though video image processing systems are quite developed, these devices usually operate by virtual loop-detectors. The advantage of this method is that the number and size of placed virtual loop-detectors can be freely set. Therefore, some spatial parameters such as spatial occupancy and density can be calculated.

In general, well-performing measurement systems can be built up by using traditional sensors (after adequate calibration and smoothing) for continuous measurement. The drawbacks are the high maintenance and installation costs that make it unrealistic to set up a system covering all important roads of a town. Another bound is that measurements take place only on separated cross sections. In other words, traditional sensors are not capable of measurements that cover a large area, e.g. OD data or travel time data cannot be obtained by them.

\subsection{Alternative road traffic measurement technologies}

Alternative sensor technology has become a real option for traffic parameter estimation due to IT developments of the last few years (Vlahogianni et al., 2014). Nowadays, numerous historic or real-time databases exist that have relevant information for road traffic parameter estimation although they have not been directly generated for traffic estimation purposes. Even though these data are not available yet for business or organizational reasons, they might be well applied for traffic control or service purposes. Expectedly, these types of data will be available soon.

One of the most common data types is the GPS data of vehicles that are part of a fleet management system. These information make real-time monitoring possible. During the operation of Floating Car Data (FCD) systems, an on-board unit provides data of the vehicle, e.g. actual speed, position or other information which are important basically for the operator, such as the fuel consumption. From these traffic data travel times, OD and route information can be estimated accurately. FCD are collected in numerous systems day-by-day either in private or public sector. In private sector usually taxis and delivery trucks apply fleet management. The system that operates in Berlin is a relevant example (Gühnemann and Scäfer, 2004). The traffic control centre is provided by real-time taxi GPS data from each vehicle in every less than 2 minutes. An example for the systems of public sector is the Hungarian 'FUTÁR' project being developed for public transportation of Budapest. Another system, operating in London, is worth to mention in which it is possible to follow real-time data for private users as well (www.tfl.gov.uk).

Another way of estimation is using Floating Mobile Data (FMD) information that contains position data of traveling mobile phones. FMD can be divided into 2 classes: client-side and server-side information systems. Client-side FMD is collected from applications typically running on smartphones for which users get traffic information in exchange. A well-known example is the Traffic application of the Google Map. The sources of data are GPS information of fleet management companies and mobile data of private smartphone users. This service does not provide exact speed values, just determines a speed category that is converted into a colour code displayed on a map for certain links (see Fig. 1).

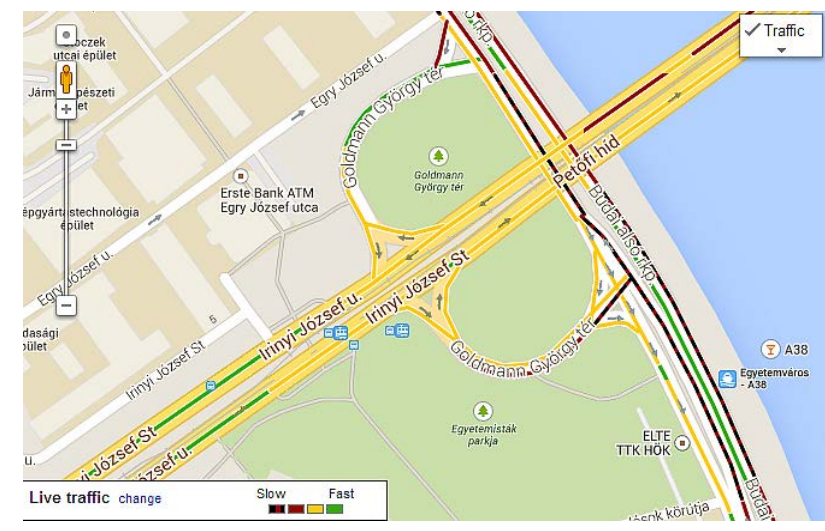

Figure 1. Google Map screenshot in Budapest 
The other way of gathering FMD information is using server-side technologies. The basis of this method is also the observation of travelling mobile phones, but only at the operator-side. If a mobile phone changes its location, it generates different types of signals according to the operation principles of radiofrequency-based telecommunication. These signals can be also applied as input data for locationbased services (Küpper, 2005). This topic, among its significant research potential in other fields, is a really important way to exploit the application of network mobile phone data for road traffic observation and management. The most considerable advantage of network data is their quantity, since nearly everyone has a mobile phone. All of the cellular signals generated by users are automatically observed by the operator through base stations. Therefore mobile phones can be treated as detectors that do not require additional development of the infrastructure. Nevertheless, processing these rough data requires special algorithms (Tettamanti and Varga, 2014). The most important advantage of server-side FMD is the measurement capability on the whole network, e.g. estimation of OD matrices. Furthermore, the estimation of velocity is also possible only by using network mobile phone data (without GPS data) Nokia Solutions and Networks OY et al., 2014).

Bluetooth-based vehicle detection is another radiofrequency-based technology that is already applied in a few cities (Quing, 2011). The system is able to estimate traffic parameters by following unique identifiers (e.g. MAC address) of wireless devices situated in vehicles.

\subsection{Features of different measurement technologies}

Beside traditional measurement techniques, even more efficient alternative ones are available for traffic parameter estimation, especially for OD matrix estimation and travel time monitoring. All the same, it is impossible to precisely estimate traffic volume on a link based on only measured speed data. Moreover, accurate cross sectional measurements cannot be used for precise estimation of network data.

So far, only the main differences have been revealed, but there are several other features by which sensor technologies can be differentiated. Tables 1, 2 and 3 show the most important factors that have to be taken into account during application (even by using more methods simultaneously).

Table 1. Features of traditional cross sectional sensors

\begin{tabular}{|l|l|}
\hline \multirow{4}{*}{$\begin{array}{l}\text { Measured and calculated } \\
\text { parameters }\end{array}$} & Volume \\
\cline { 2 - 2 } & time occupancy \\
\cline { 2 - 2 } Cycle time & velocity \\
\cline { 2 - 2 } Technical advantages & time headway \\
\hline \multirow{5}{*}{ Drawbacks } & optional \\
\hline & reliability of measurements \\
\cline { 2 - 2 } & availability \\
\cline { 2 - 2 } & high installation and maintenance costs \\
\cline { 2 - 2 } & not capable to cover whole networks due to high costs \\
\cline { 2 - 2 } & not capable to extend measurements (OD matrix, travel times routes) \\
\cline { 2 - 2 } & frequent breakdowns \\
\hline
\end{tabular}

Table 2. Features of FCD and client-side FMD sensors

\begin{tabular}{|l|l|}
\hline \multirow{4}{*}{$\begin{array}{l}\text { Measured and calculated } \\
\text { parameters }\end{array}$} & position with timestamp \\
\cline { 2 - 2 } & velocity \\
\cline { 2 - 2 } & travel time \\
\cline { 2 - 2 } & route \\
\cline { 2 - 2 } Cycle time & OD information \\
\hline \multirow{3}{*}{ Technical advantages } & system-dependent \\
\hline \multirow{4}{*}{ Drawbacks } & reliability of measurements \\
\cline { 2 - 2 } & no installation and maintenance costs, data can be purchased as a service \\
\hline & $\begin{array}{l}\text { available FCD information are not necessarily representative because of the quantity } \\
\text { (FCD vehicle are not everywhere at every time) }\end{array}$ \\
\cline { 2 - 2 } & estimating the traffic state of a whole network requires data of more fleets \\
\cline { 2 - 2 } & not capable to calculate traffic volume data \\
\cline { 2 - 2 } & limited application of data from taxis and buses moving in bus lanes \\
\hline
\end{tabular}


Table 3. Features of server-side FMD sensors

\begin{tabular}{|l|l|}
\hline \multirow{4}{*}{$\begin{array}{l}\text { Measured and calculated } \\
\text { parameters }\end{array}$} & position with cell-identifier and timestamp \\
\cline { 2 - 3 } & travel time \\
\cline { 2 - 2 } Cycle time & route \\
\cline { 2 - 2 } Technical advantages & OD information \\
\hline \multirow{5}{*}{ Drawbacks } & optional \\
\hline & $\begin{array}{l}\text { available FCD information are representative because of the quantity (almost every } \\
\text { traveler has a mobile phone) }\end{array}$ \\
\cline { 2 - 2 } & capable to estimate the OD matrix of the whole network \\
\cline { 2 - 2 } & no installation and maintenance costs, data can be purchased as a service \\
\hline & route information can only be estimated \\
\cline { 2 - 2 } & $\begin{array}{l}\text { travel time data are inaccurate for short trips because locations of handovers have a } \\
\text { standard deviation, but during longer trips the error becomes smaller }\end{array}$ \\
\cline { 2 - 2 } & in non-urban regions the estimation is not as accurate due to bigger cell sizes \\
\cline { 2 - 2 } & $\begin{array}{l}\text { data generated by travelers using public and private transport have to be separated by } \\
\text { smoothing from a database containing all incoming data }\end{array}$ \\
\hline
\end{tabular}

Table 4 gives a short review of traffic data that can be measured or estimated by sensor technologies.

Table 4. Classification of traffic data

\begin{tabular}{|l|l|l|}
\hline Measurement & Event based & Aggregated \\
\hline Cross sectional & current velocity, occupancy, time headway & $\begin{array}{l}\text { time mean speed, volume, time mean } \\
\text { headway }\end{array}$ \\
\hline Spatial & $\begin{array}{l}\text { travel time, mean velocity, position with } \\
\text { timestamp, vehicle trajectory }\end{array}$ & $\begin{array}{l}\text { space mean speed, density, OD matrix, mean } \\
\text { travel time }\end{array}$ \\
\hline
\end{tabular}

\section{Integrated use of different measurement systems}

The advantages and disadvantages of different sensors have been described in Section 2. The purpose of combined sensor technologies is based on the opportunity that fusion of different data types results in a better outcome than using only one type of sensor data.

\subsection{Data fusion}

Data fusion is not a specific technique, just an object of making an integrated database of different types of information. (Mitchell, 2007) defines data fusion as follows: 'The theory, techniques and tools which are used for combining sensor data, or data derived from sensory data, into a common representational format. In performing sensor fusion our aim is to improve the quality of the information, so that it is, in some sense, better than would be possible if the data sources were used individually.'

Certainly, data fusion is not always necessary. If a perfect information source existed, there would be no need to use this technique. Using data fusion techniques may have the following advantages (Bachmann, 2011), (Klein and Mills, 2006), (Ng, 2003):

- Reliability/Robustness/Redundancy: 'The system fusing several sources of data has a higher fault-tolerance.'

- Accuracy/Certainty: 'The combination of several different kinds of sensors can produce more accurate information.'

- Completeness/Coverage/Complementarity: 'More data sources will provide extended coverage of information on an observed object or state.'

- Cost effectiveness: 'If using sensors on the whole network, especially on a traffic network, was too expensive, applying data fusion techniques would be a profitable alternative.'

- Representation: 'Sensor fusion is necessary to combine information and clearly present the best interpretation of the sensor data.'

- Timeliness: 'More timely information may be provided by multiple sensors.'

\subsection{The purpose of data fusion in transportation}

Measurement, information and control systems cannot reach maximal effectiveness if they work separately, because none of them has information on the entire network. If rough data is processed in an integrated way, the information about the network is more precise that results in the fact that all subsystems work in a more efficient way. This means that data fusion can help all intelligent 
transportation subsystems to improve (Anand et al., 2013), (El Faouzi et al., 2011). The functions of these subsystems are usually measurement, estimation, forecasting, control and information collecting or providing (Quing, 2011).

- Measurement systems: Data fusion techniques make it possible to make a standard platform for data having different semantics and syntax. The next step is the development of estimating and data completing algorithms that can also analyze those parts of the network from which in a current time step no sensor information have been collected.

- Information systems: Providing information for travelers and drivers is a basic criterion of transportation nowadays. Modern navigation systems are provided with traffic and road state information. Certainly, the knowledge of the entire network state is an advantage.

- Estimation and forecast: The knowledge of the present state of the network is crucial for traffic control. The result can be even better if not only is the present state of the network known, but the future state can also be forecast (Guo et al., 2013).

- Network traffic control: Besides providing information for travelers, improving the efficiency of navigation and incident detection, the most important factor of traffic data fusion is providing data for traffic network control. In this case the state of traffic can be optimized based on traffic volume and OD data.

Certainly, these systems do not always operate separately. Moreover, they cannot often be separated, since the systems support or complete each other in many cases.

\subsection{Data fusion methods in road transportation}

Many articles have been written recently that have introduced different data fusion techniques. The purpose of this section is to give a short review of them.

The most common used data fusion technique among researchers is the Kalman Filter (Wardrop, 1952), (Welch and Bishop, 1995) with its variants (e.g. the extended Kalman Filter). The first time it was applied for road traffic was in 1972 by Szeto and Gazis (Szeto and Gazis, 1972). The basic theory of this research is that most analytic traffic models can be generated by state space representation. One of these solutions is described in (Chu et al., 2005) claiming that even only measuring by more loop-detectors can meet the requirements of sensor fusion. All the same, some other researches use inhomogeneous data sources to improve estimation quality. In (Herrera and Bayern, 2007) the traditional cross sectional measurement is combined with moving sensors that actually means following GPS based trajectories of some specific vehicles. In this case from GPS data of moving vehicles, by using the conservation equation and the macroscopic model, density and speed of a specific location can be determined. That has a same representation as the cross sectional measurements.

The most considerable advantage of the application of different digital filters is the fact that estimation can be done in an iterative way, whereas capacity requirements of computation are low. As the capacity limit of computations had extremely increased, new real-time solutions became available, which is the reason for optimizing techniques being widespread. The target is to compose an objective function for a specific traffic data, e.g. for travel time. Constraints are given by traffic models and the data themselves. This method is used in (Böker and Lunze, 2002) in which cross sectional measurements vehicle measurements and the traffic model are combined. These give constraints for linear programming equations of which the solutions are the estimated minimum or maximum travel times.

Another way of traffic parameter estimation with low computing capacity is represented by interpolation techniques. These are similar to convolution image filtering techniques. A smoothing convolutional filter technique was introduced in (Treiber and Helbing, 2002) by using the interpolation technique in order to fuse stationary data (loop-detectors) and non-stationary (FCD) measurements.

Previous techniques exploit the relationship between the measured data and the traffic model. During using data fusion techniques, by weighting the traffic model and the measured data, a balance can be set among them. Another method is suggested by (Quing, 2011) using the principle of data-data consistency. It calibrates one or two pieces of measured data with each other by using only non-linear models. Smoothing the noise of loop-detector data by comparison with noiseless FCD based travel time data is a typical example for this method.

\section{A data fusion method for urban road traffic}

Data fusion is one of the most researched topics in transportation due to the fast improvement of information technology. Techniques introduced in scientific articles are usually to reconstruct the state of 
motorway traffic by using cross sectional measurements (e.g. loop-detector) and FCD/FMD (e.g. Claudel and Bayern, 2008). The opportunity of using cell information of mobile phone networks in transportation is an unexploited research field at present. Another unexploited research field is the construction of an integrated data model that can estimate the traffic state for an entire urban network by using all sensor measurements. Thus, there is a great potential in these research fields. Therefore, in the following part of the article the basis of an integrated data fusion model for urban traffic data are described. This research has been invoked by the 'Smarter Transport' project (TÁMOP-4.2.2.C-11/1/KONV-2012-0012) of which the purpose is the development of a traffic data collecting technology based on combined measurement systems.

\subsection{Problems of the urban network}

The application of a data fusion method on an urban network has several difficulties caused by the features of urban traffic. An obvious problem is the complicated road network, so route reproduction from server-side FMD sensor data is much more difficult compared to motorways.

Another difficulty is the effect of signalized traffic control that has a strong influence on traffic flow. Traffic on motorways is described by macroscopic fluid models that have a clear principle. In contrast, urban models are either rough or even if they are detailed, macroscopic models often cannot be applied because of the high number of necessary measurements and computation.

A further problem is that only loop-detector is capable of direct precise traffic volume measurement, all of the other sensors are not. The OD data of server-side FMD can be applied only for a rough estimation, e.g. estimation of aggregated zone-to-zone travel data.

Considering the problems, the purpose of this research was to find an approach that does not use complicated models and only tries to precisely estimate the traffic state of specific urban roads by the fusion of different types of sensor data. Since FMD/FCD measurements represent travel time well and it can also be estimated from loop-detector measurements, the unknown parameter of the integrated data fusion model is travel time.

\subsection{Kalman Filter based sensor fusion}

The proposed data fusion methodology is based on the method of the Kalman Filter estimation often used by traffic engineers. This technique is a recursive method for linear filtering of discrete data (Welch and Bishop, 1995). The method makes it possible to precisely estimate the state of a dynamic system, even if its exact nature is unknown.

The linear Kalman Filter can be applied if the state space representation of the system is determined. The state of a discrete, sampled, time-invariant process is described with a linear difference equation

$x(k+1)=A x(k)+B u(k)+w(k)$,

where $x(k) \in \mathbb{R}^{n}$ is the state vector, $u(k) \in \mathbb{R}^{m}$ is the control input vector, $w(k)$ is the process noise, $A \in \mathbb{R}^{n \times n}$ and $B \in \mathbb{R}^{n \times m}$ coefficient matrices and $k=1,2, \ldots$ is the discrete timestep. $x(k+1)$ describes the state change in the $[k T,(k+1) T]$ time interval, where $T$ is the sampling time period. The measurement equation of the system generally is:

$y(k)=C x(k)+v(k)$,

where $y(k) \in \mathbb{R}^{p}$ is the vector containing measurement data, $v(k)$ is the process noise and $C \in \mathbb{R}^{p \times n}$ is the measurement matrix. The filter can be applied if process noise $w(k)$ and measurement noise $v(k)$ are assumed to be zero mean Gaussian white noise, so $E\{w(k)\}=0$ and $E\{v(k)\}=0$. The covariance matrices are assumed to be

$Q=E\left\{w(k) w(k)^{T}\right\}$

$R=E\left\{v(k) v(k)^{T}\right\}$

The Kalman Filter estimates the state of the next time step based on the state of the current time step but the estimation is refined in the next time step considering the measurement result of the period. Furthermore, it estimates system covariance that is also refined by the result of the latest measurement. It means that the filter has a two-phase operation: prediction and correction. Let $\hat{x}^{-}(k)$ be the a priori state estimate at step $k$ and $\hat{x}(k)$ the a posteriori state estimate at step $k$. Now, the a priori and a posteriori 
estimate errors are defined which make it possible to determine the a priori and a posteriori estimate covariance $P^{-}(k)$ and $P(k)$ which represent the square of the estimated errors. The two-phase estimation algorithm is shown in Fig. 2.
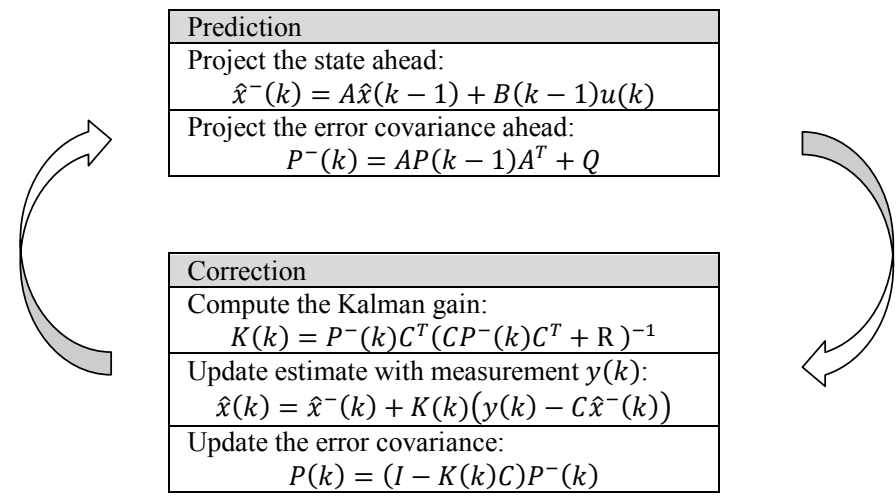

Figure 2. The two-phase Kalman Filter algorithm

The Kalman Filter introduced so far can be directly applied for sensor fusion. The only condition is that correlation among different measurement noises is not permitted.

If the results of sensor measurements are temporarily available, the state space representation of travel time estimation is:

$x(k+1)=x(k)+w(k)$,

$y(k)=C x(k)+v(k)$.

where (5) describes the system dynamics based on the random walk model, that is $A=I$ and $B=0$. The reason for it is the unknown changing behaviour of travel time, therefore it is considered as a random process. Since in this example travel time is estimated only for a single link, $x(k)$ and $w(k)$ are scalar variables. At the same time, $y(k)$ in the measurement equation describes a system of equations. The dimension of $y(k)$ equals the number of sensors. For example, if 2 different sensors (e.g. FCD and FMD) are involved measuring travel time:

$\left[\begin{array}{l}y_{1}(k) \\ y_{2}(k)\end{array}\right]=\left[\begin{array}{l}1 \\ 1\end{array}\right] x(k)+\left[\begin{array}{l}v_{1}(k) \\ v_{2}(k)\end{array}\right]$

The Kalman Filter can estimate state directly from measurements $y_{1}(k)$ and $y_{2}(k)$ that are automatically weighed according to their covariance. It is followed by their fusion that results in an integrated state estimation. The more precisely the standard deviations $v_{1}(k)=\sigma_{1}$ and $v_{2}(k)=\sigma_{2}$ are known, the more precise the operation is. Standard deviations can be determined in an empirical way. In the example they can be calculated by the standard deviations of general FMD and FCD measurements.

\subsection{Sensor fusion with the Switching Kalman Filter}

The sensor fusion methodology introduced in the previous subsection can be applied easily and efficiently if synchronized measurement data can be gathered at each measurement period. This condition, however, is not always fulfilled even on urban roads with heavy traffic. A possible solution for the problem is the application of the switching system state space representation (Liberzon, 2003). In this case, the system is described with more (but not infinite) space state representations that practically mean different operational modes. Moreover, different Kalman Filters can be constructed for different operational modes. The system changes its operational modes followed by the Kalman Filter switching into another mode as a reaction for the change of external effects (Böker and Lunze, 2002). The switching, discrete and linear state space representation based on (1) and (2):

$x(k+1)=A_{\rho(k)} x(k)+B_{\rho(k)} u_{\rho(k)}(k)+w_{\rho(k)}(k)$,

$y_{\rho(k)}(k)=C_{\rho(k)} x(k)+v_{\rho(k)}(k)$, 
$\rho(k) \in S=\{1,2, \ldots, s\}$,

where $\rho(k)$ is the discrete switching signal that refers to the operational mode of the system in time step $k T$ and determines the values of matrices $A, B, C$ and signals $u, w, v$. Considering its temporal states, $\rho(k)$ can be rule-based or a random sequence signal.

The operation of the Switching Kalman Filter is based on the algorithm shown in Fig. 2, the difference is that in this case the algorithm is always actualized for the current $\rho(k)$ system mode. The filter contains intermediate values $\hat{x}^{-}(k)$ and $P^{-}(k)$, even if the current mode is different from the previous one, i.e. $\rho(k) \neq \rho(k-1)$. The modified estimation algorithm is shown in Fig. 3 .

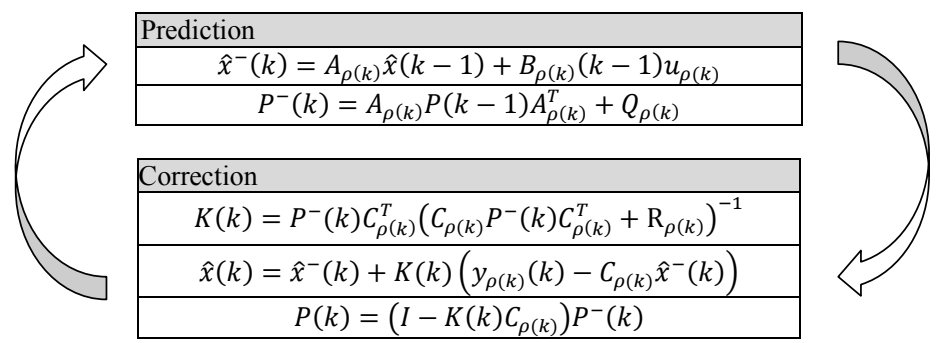

Figure 3. The two-phase algorithm of the Switching Kalman Filter

\subsection{Travel time estimation on urban links by sensor fusion}

Using the Switching Kalman Filter is a solution for the problem of inhomogeneous sensors producing inhomogeneous data during road traffic measurements. Therefore, the continuously changing number of sensors does not cause any difficulties, at the same time the benefits of Kalman Filter can be exploited for data fusion. The purpose of this subsection is to describe a data fusion methodology for the estimation of travel time on any urban link, exploiting the opportunities.

The Switching Kalman Filter can be applied if the system can be observed, that is:

$\operatorname{rank}\left[\begin{array}{c}C_{\rho(k)} \\ C_{\rho(k)} A_{\rho(k)} \\ \vdots \\ C_{\rho(k)} A_{\rho(k)}^{n-1}\end{array}\right]=n$.

If no measurement data is available for a current time step, this requirement is not met. If the data of at least one sensor is at service, the requirement is met. Considering the frequency of sensor measurements and the number of sensors, only spatially fixed sensors are able to measure continuously by all means. As a consequence, at least one cross sectional loop-detector is required. In urban networks this might be usually available. Any other sensors (e.g. FCD, FMD, Bluetooth, etc.) are considered as potential devices in the network, whereas their permanent operation is not a requirement. The main drawback of this approach is measuring travel times by cross sectional detectors, which obviously cannot be done directly. Therefore simulations have been done in a modelled environment in which it is possible to determine the mean travel time on a link within typical traffic volume conditions. The result can be either a function $(T=f(Q))$ or a simple determination of volume scales. In that case, as an example, a mean travel time can be determined for $0-200 \mathrm{veh} / \mathrm{h}$, another one for $201-500 \mathrm{veh} / \mathrm{h}$ and so on. Travel times determined for links within different volume conditions during simulations can be applied as measurement results. For a volume data measured by a real detector, a simulation-based mean travel time can be assigned.

The applied switching system based on (5) and (6):

$x(k+1)=x(k)+w(k)$,

$y_{\rho(k)}(k)=C_{\rho(k)} x(k)+v_{\rho(k)}(k)$,

$\rho(k) \in S=\{1,2,3,4\}$,

where state variable $x(k)$ represents mean travel time of a specific link. Switching signal $\rho(k)$ has an effect only on the measurement equation. Set $S$ contains four possible different measurement combinations using 3 different sensor types (loop-detector, FMD, FCD). The combinations are shown in Table 5. 
Table 5. The different measurement configurations

\begin{tabular}{|c|c|c|c|}
\hline $\boldsymbol{\rho}(\boldsymbol{k})$ & $\boldsymbol{y}_{\boldsymbol{\rho}(\boldsymbol{k})}(\boldsymbol{k})$ & $\boldsymbol{C}_{\boldsymbol{\rho}(\boldsymbol{k})}$ & $\boldsymbol{v}_{\boldsymbol{\rho}(\boldsymbol{k})}(\boldsymbol{k})$ \\
\hline 1 & {$\left[T_{1}^{\text {loop }}\right]$} & {$[1]$} & {$\left[\sigma_{1}\right]$} \\
\hline 2 & {$\left[\begin{array}{l}T_{1}^{l o o p} \\
T_{2}^{F C D}\end{array}\right]$} & {$\left[\begin{array}{l}1 \\
1\end{array}\right]$} & {$\left[\begin{array}{l}\sigma_{1} \\
\sigma_{2}\end{array}\right]$} \\
\hline 3 & {$\left[\begin{array}{l}T_{1}^{l o o p} \\
T_{3}^{F M D}\end{array}\right]$} & {$\left[\begin{array}{l}1 \\
1\end{array}\right]$} & {$\left[\begin{array}{l}\sigma_{1} \\
\sigma_{3}\end{array}\right]$} \\
\hline 4 & {$\left[\begin{array}{l}T_{1}^{l o o p} \\
T_{2}^{F C D} \\
T_{3}^{F M D}\end{array}\right]$} & {$\left[\begin{array}{l}1 \\
1 \\
1\end{array}\right]$} & {$\left[\begin{array}{l}\sigma_{1} \\
\sigma_{2} \\
\sigma_{3}\end{array}\right]$} \\
\hline
\end{tabular}

Measurement vector $y_{\rho(k)}(k)$ contains the combination of travel times measured by sensors of different types. Note that the first element of $C_{\rho(k)}$ is always 1, which represents permanent loop-detector measurements, hence satisfies Eq. (11). The second and third element can be either 1 or 0 . If no FCD and/or FMD have been detected within the current time step, the value is 0, else 1. The Switching Kalman Filter switches according to the signal $\rho(k)$ (see Table 5) that is a known value.

For a precise estimation measurement noise $v_{\rho(k)}(k)$ is assumed to be known. This can be determined by the standard deviation of measurements. The values of $v_{\rho(k)}(k)$ continuously change, since the standard deviation of a sensor is not temporary like FCD based travel time if it is a result of interpolation. The standard deviation of loop-detector data can be calculated by the evaluation of simulation runs. Standard deviation of travel times measured by FCD and FMD techniques can change even within the current measurement. Travel times calculated by the cross sectional detector have the highest standard deviation. The noise of FCD/FMD measurements is much lower, which results in more precise estimations. As a consequence, measurement estimation is expected to be much reliable during the rush hours (when FCD/FMD are probably available) and less out of them. However, during these periods travel time data can be calculated from static databases, since the effect of traffic is low. The described data fusion estimation method is shown in Fig. 4.

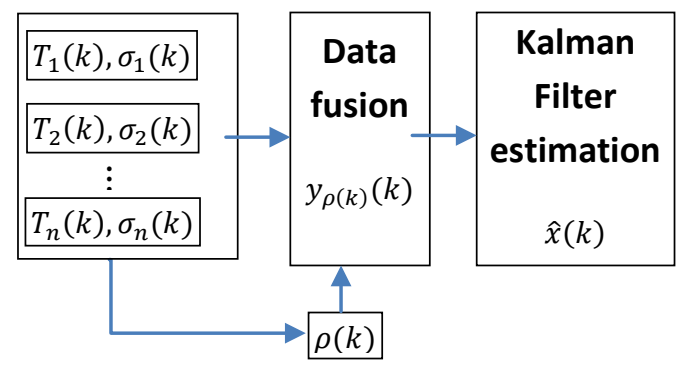

Figure 4. The sensor fusion method for the estimation of mean travel times on urban links

\subsection{Sensor fusion for network traffic estimation}

Information produced by the method introduced in the last subsection would be useful for travelers. All the same, these estimation data can be applied for the entire network as well. Therefore they can be used for the refinement of macroscopic traffic estimation. In a previous article of the authors (Tettamanti and Varga, 2014), route assignment has been developed by using travel time data. If either a predetermined (using historical data) or a real-time (using cellular data) estimated OD matrix is available, traffic can be assigned in a traditional way (Wardrop, 1952). Practically, the assignment creates a traffic model estimating traffic volume on the network links. This method can be improved by involving travel time results of the data fusion methodology. Hereby, a dynamic traffic assignment can be done applying the real-time traffic data.

The main point of the method is that performance functions $\left(t_{a}\right)$ of links are limited by the measured travel time data (Tettamanti and Varga, 2014). Therefore optimizing traffic assignment has another bound:

$t_{a}^{m}\left(1-\Delta_{a}\right) \leq t_{a} \leq t_{a}^{m}\left(1+\Delta_{a}\right)$,

where $t_{a}^{m}$ represents the measured mean travel time for a link and $\Delta_{a}$ is an uncertainty factor that can be empirically calibrated for example by the standard deviation of the measurement. 


\subsection{Difficulties of real-world application}

The opportunities of the introduced measurement methodologies are described in the previous sections. In this subsection the difficulties of real-world application are analysed.

The biggest uncertainty of server-side measurements is the error of mobile phone location. Location area updates and handovers are not absolutely deterministic; therefore the location of such events can have a standard deviation up to hundreds of meters. Thus, these types of measurement techniques are worth using for longer trips, where the degree of standard deviation compared to the trip length is low.

In general, another serious problem of FMD measurements is that the travel mode is unknown; therefore pre-filtering is required in order to differentiate motorists from pedestrians.

However, FCD are definitely vehicle data, some vehicle classes have special 'disturbing' features. For example taxis and buses can use bus lanes, the velocity of bigger vehicles is lower, delivery vehicles often stop on the road for loading and unloading, etc.

Considering these factors, it is obvious that the method introduced in this article has many factors to be solved but none of them is impossible from engineering aspect.

\section{Conclusions}

In this article the applied methods of traffic measurements and their classification including some of the sensor technologies have been revised. After that, a data estimation concept has been introduced for urban network traffic. The proposed methodology using sensor fusion technology is based on the Switching Kalman Filter that makes the efficient integrated application of very inhomogeneous types of sensor data possible. Moreover, the method has another opportunity, since using data provided by data fusion can improve real-time macroscopic traffic modelling.

The future purpose of the research is the validation of the introduced methodology by simulation runs and real data. The research is related to a Hungarian town, Györ. Accordingly, using sensor data from the network of the town is planned.

\section{Acknowledgements}

This work is connected to the scientific program of EITKIC-12-1-2012-0001 project (supported by the Hungarian Government, managed by the National Development Agency, and financed by the Research and Technology Innovation Fund), and TÁMOP-4.2.2.C-11/1/KONV-2012-0012: Smarter Transport project (supported by the Hungarian Government and co-financed by the European Social Fund).

\section{References}

1. Anand, A., Ramadurai, G. and Vanajakshi, L. (2013). Data Fusion Based Traffic Density Estimation and Prediction, Journal of Intelligent Transportation Systems: Technology, Planning, and Operations, accepted author version, DOI: 10.1080/15472450.2013.806844

2. Bachmann, C. (2011). Multi-Sensor Data Fusion for Traffic Speed and Travel Time Estimation, MSc Thesis, Toronto

3. Böker, G., Lunze, J. (2002). Stability and performance of switching Kalman filters, International Journal of Control, 75(16/17): 1269-1281

4. Claudel, C. G., Bayen, A. M. (2008). Guaranteed bounds for traffic flow parameters estimation using mixed lagrangian-eulerian sensing. Proceedings of the 46th Annual Allerton Conference on Communication, Control, and Computing, Allerton, IL

5. Chu, L., Oh, J., Recker, W. (2005). Adaptive Kalman filter based freeway travel time estimation, 84th TRB Annual Meeting, Washington

6. El Faouzi, N-E., Leung, H., Kurian, A. (2011). Data fusion in intelligent transportation systems: Progress and challenges - A survey, Information Fusion, 12(1): 4-10 DOI: 10.1016/j.inffus.2010.06.001

7. Guo, F., Krishnan R. and Polak, J. (2013). A computationally efficient two-stage method for shortterm traffic prediction on urban roads, Transportation Planning and Technology, 36(1): 62-75, DOI:10.1080/03081060.2012.745721 
8. Gühnemann, A., Schäfer, R. P. Thiessenhusen, K. U., Wagner, P. (2004). Monitoring Traffic and Emissions by Floating Car Data, ITS Working Paper ITS-WP-04-07, Institute of Transport Studies, University of Sydney

9. Herrera, J. C., Bayen, A. M. (2007). Traffic flow reconstruction using mobile sensors and loop detector data, 87th TRB Annual Meeting

10. Klein, L. A., Mills, M. K. (2006). Gibson, D. R. P.: Traffic Detector Handbook:Third Edition, Federal Highway Administration Turner-Fairbank Highway Research Center

11. Küpper, A. (2005). Location-based Services. John Wiley \& Sons, ISBN 978-0470-09231-6.

12. Liberzon, D. (2003). Switching Systems and Control, Birkhäuser, Boston

13. Ludvig, Á., Tettamanti, T., Varga, I. (2012). Travel time estimation in urban road traffic networks based on radio signaling data. In: 14th International Conference on Modern Information Technology in the Innovation Processes of Industrial Enterprises, MITIP, Budapest,2012.10.24-2012.10.26. pp. 514-527. ISBN: 978-963-311-373-8

14. Nokia Solutions and Networks OY, Vékony, N., Tettamanti, T, Varga, I., Ludvig, Á. (2014) Determining travel information (patent), Invention Publication No. WO/2014/023339012E00798 HU, http://patentscope.wipo.int/search/en/WO2014023339

15. Mitchell, H. B. (2007). Multi-sensor data fusion: An introduction, New York, Springer

16. Ng, G. W. (2003). Intelligent systems - fusion, tracking and control, Philadelphia: Research Studies Press Ltd.

17. Qing, O. (2011). Fusing Heterogeneous Traffic Data: Parsimonious Approaches using Data-Data Consistency, PhD Thesis, Delft University of Technology

18. Szeto, M. W. - Gazis D. C. (1972). Application of Kalman Filtering to the Surveillance and Control of Traffic Systems, Transportation Science, 6(4):419-439.

19. Tettamanti T., Varga I. (2014). Mobile phone location area based traffic flow estimation in urban road traffic, Columbia International Publishing, Advances in Civil and Environmental Engineering, 1(1):1-15.

20. Transport for London website: Developers' Area http://www.tfl.gov.uk/businessandpartners/syndication/16492.aspx

21. Treiber, M., Helbing, D. (2002). Reconstructing the spatio-temporal traffic dynamics from stationary detector data, Cooperative Transportation Dynamics, 1(3):1-24.

22. Vlahogianni, E., Karlaftis, M. G., Golias, J. C. (2014). Short-term traffic forecasting: Where we are and where we're going, Transportation Research Part C (2014), in press, DOI: $10.1016 / j . t r c .2014 .01 .005$

23. Wardrop, J. G. (1952). Some Theoretical Aspects of Road Traffic Research. Proceedings of the Institute of Civil Engineers, 1-2(9):325-378.

24. Welch, G., Bishop, G. (1995). An Introduction to the Kalman Filter, University of North Carolina at Chapel Hill, TR95-041 